FINAL REPORT

\title{
DIFFERENTIAL GROUP-VELOCITY DETECTION OF FLUID PATHS (SUPPLEMENTAL REQUEST)
}

Project Director: Leland Timothy Long

\author{
Award to: Georgia Tech Research Corporation \\ School of Earth and Atmospheric Sciences \\ Georgia Institute of Technology \\ Atlanta, Georgia 30332-0340
}

PeopleSoft Project Number: 3506C20

Project Number: G-35-C20

Award Number: DE-FG02-04ER63866

US DEPT OF ENERGY/ARGONNE, IL

This material is based upon work supported by the U.S. Department of Energy under Awards Number DE-FG02-04ER63866 and Number DE-FG01-04ER04-02

Disclaimer: Any opinions, findings, and conclusions or recommendations expressed in this material are those of the author(s) and do not necessarily reflect the views of the Department of Energy.

Distribution is unlimited 


\section{DIFFERENTIAL GROUP-VELOCITY DETECTION OF FLUID PATHS}

\section{EXECUTIVE SUMMARY}

For nearly 50 years, surface waves that propagate through near-surface soils have been utilized in engineering for the determination of the small-strain dynamic properties of soils. These techniques, although useful, have not been sufficiently precise to use in detecting the subtle changes in soil properties that accompany short-term changes in fluid content. The differential techniques developed in this research now make it possible to monitor small changes (less than $3 \mathrm{~cm}$ ) in the water level of shallow soil aquifers. Using inversion techniques and tomography, differential seismic techniques could track the water level distribution in aquifers with water being pumped in or out. Differential surface wave analysis could lead to new ways to monitor reservoir levels and verify hydrologic models. Field data obtained during this investigation have measured changes in surface-wave phase and group velocity before and after major rain events, and have detected subtle changes associated with pumping water into an aquifer and pumping water out of an aquifer. This research has established analysis techniques for observing these changes. These techniques combine time domain measurements to isolate surface wave arrivals with frequency domain techniques to determine the effects as a function of frequency. Understanding the differences in response as a function of wave frequency facilitates the inversion of this data for soil velocity structure. These techniques have also quantified many aspects of data acquisition and analysis that are important for significant results. These include tight control on the character of the source and proper placement of the geophones. One important application is the possibility that surface waves could be used to monitor and/or track fluid movement during clean-up operations, verifying that the fluid reached all affected areas. Extending this to a larger scale could facilitate monitoring of water resources in basins without having to drill many expensive wells. The next step is to investigate the commercial applications of differential surface wave analysis. 


\section{DIFFERENTIAL GROUP-VELOCITY DETECTION OF FLUID PATHS}

\section{SUMMARY OF PROJECT PLANS AND ACCOMPLISHMENTS}

This project is a supplement to a two-year project. The results during the first year were encouraging and we considered it appropriate to polish the analysis and expand the number of test sites.

The stated objectives and accomplishments are as follows:

Objective 1. During the remainder of the second year we will continue to develop the techniques to compare surface-wave records and compute the differences in dispersion for inversion

The analysis technique has been significantly modified to solve many sources of error in the data. A new dispersion inversion program was written to incorporate a velocity gradient in the solution, instead of the conventional flat layers.

Objective 2. The inversion program for velocity structure will be put together the summer of 2004 and applied to the new refraction line data sets.

This was accomplished as a special project to convert the many components of the inversion program into a single coherent sequence of programs. Michael Chen (Chen, 2004) presented this work as a conference talk.

Objective 3. During the rest of the second year and the third year we plan to apply the technique to the Panola Mountain Research Watershed test area where perturbations in water content can be monitored during multiple seismic tests under varying water saturation conditions (following rainstorm events) and evaluated for repeatability.

Over 6 data sets were obtained at Panola Mountain. Two were after significant rain events. During one test, data were obtained at one-minute intervals while pumping water into the aquifer below the seismic line. The analyses of these data are described below.

Objective 4. New sites in differing geologic settings will be tested.

We obtained for analysis a data set at the Georgia Southern University research well site. The geology there is the coastal plane sands, which act as a shallow aquifer. Two other sites were tested, one with hard rock and the other with a thin soil overburden on hard rock to initiate the tests related to scattering from fractures with varying amounts of water content. These sites did not yield useful differential seismic data, but the scattering inversion did image the fracture zones. The analysis of this data has developed into the Ph.D. thesis topic of Tatiana Toteva.

Objective 5. Finally, during the third year the differential technique will be tested on a set of surface-wave tomography data covering an area of perturbation.

The Georgia Southern University research well site was designed as a tomography experiment. Data were obtained before and after pumping out the well. The analysis is described below. 
Objective 6. The results of the study are to be presented at a number of meetings and in 3 to 5 publications based on those presentations over the next year and a half.

These are listed below under technology transfer activities. The major contributions of this research will be presented at SAGEEP, 2006, in Seattle and placed into three papers in preparation. 


\section{SUMMARY OF PROJECT ACTIVITIES FOR PERIOD OF FUNDING.}

Original hypothesis: This research was to develop and test a new technology to aid in the selection and design of remediation options in shallow aquifers. The objective of the new technology is to map the primary fluid pathways in shallow soils. We proposed to measure perturbations in the shear wave velocity of the soil by measuring changes in seismic response either induced by natural processes, (e.g. rainfall) or induced actively by pumping and withdrawal of fluids. The observed changes in surface-wave velocity will then be used to map areas where fluids modified the fluid pressure and, hence, the shear-wave velocity. The proposed technique should identify areas reached by the fluids under normal or induced flow patterns. The proposed technique takes advantage of the abilities of signal-processing techniques to detect small differences in propagation. The differences should be sensitive to flow paths relative to a reference structure that need only approximate the actual structure. In effect, we propose an inversion of the perturbation of surface wave velocity instead of the inversion of surface wave dispersion for structure.

We expect the differential measurements to provide significantly better precision than is possible with the direct estimation of the structure from a dispersion curve. The perturbations in the velocity structure will indicate where, in a time sequence, the pumped fluids have influenced the shear-wave velocity. Areas experiencing the greatest pressure and material property changes would indicate primary flow paths and zones that are most likely amenable to remediation by extraction or flushing. A time sequence of tests could monitor the dispersion and movement of the induced perturbations. The technique could be used prior to remediation to detect flow paths and, hence, help design an optimal remediation process. It could be used during remediation to detect and determine which zones are reached by the remediation. The proposed technique is similar to techniques being developed in the oil industry to monitor depletion of oil reserves during pumping for secondary and tertiary recovery operations in an oil field.

Approaches used: The engineering techniques applied to surface waves are in effect only different ways to define a dispersion relation. Consequently, they all reduce to the same fundamental problem, which is to find the structure that predicts an observed surface wave dispersion relation. The determination of structure from a dispersion curve is a non-linear problem that is solved by successive approximations. In general, the lack of precision and occasional lack of stability of inversion methods for dispersion curves does not allow easy evaluation of subtle differences in structure that might be introduced by fluid injection or withdrawal. Also, the resolution of structures using surface waves may be limited by interference by signals from other phases and noise.

The original proposal was to use the Finite Difference technique as a theoretical source of waveforms in the analysis so that solution will be sensitive to relative excitation of higher mode surface waves, to the mixture of modes at any given time in the trace, to the effects of a velocity gradient on the character of wave propagation and to the conversion of surface waves to body waves at boundaries. However, the mixed modes and arrivals made reliable phase velocity measurements

difficult. We now propose to use a combination of techniques. The Finite Difference 
modeling will still be use to assess the impact of heterogeneity in velocity structure. However, the dispersion relations will be generated using the gradient formulation of the solution to the equations of motion, as proposed by Takeuchi, H., and Saito, 1972.

The object of this research has been to investigate the ability of differential techniques to map permeable areas by looking for and modeling temporal variations in shear-wave velocity. Instead of mapping variations in structure (note, the structure could be provided as a separate analysis) we look for time variations in the dispersion curves (as indicated by time variations in the seismic trace) induced by changes in fluid pressure. The reference or average structure is no longer critical to the analysis so long as a reasonable approximation is available to generate appropriate theoretical differences in dispersion induced by known perturbations in the structure. For the differential approach, the reference is not the pre-test velocity structure, which is generally unknown in detail, but is the seismic trace from the unperturbed structure, which is measured in detail. In the differential approach, we propose to measure the differences in the dispersion for waves traveling the same path by direct comparison of two seismic traces recorded at different times. The reference used in the differential approach becomes the trace obtained during a steady-state condition. We next use the deviations to map the areas that are influenced by perturbations in the fluid concentration or hydrostatic pressure. For an analysis of the perturbations, it is not necessary to know the exact structure. So long as a reasonably representative base model is available, we can generate relations between perturbations in dispersion and perturbations in structure. It is only necessary to know how the dispersion curves are perturbed.

Differential techniques may be applied to surface-wave group-velocity tomography or to phase-velocity measurements. In group-velocity tomography, the multiple-filter outputs from two traces obtained at two different times would be compared to estimate differences in the group arrival times. Then tomography is used to invert the differences in arrival time for differential group velocity dispersion curves at each pixel. These differential group-velocity dispersion curves are then inverted for perturbations in shear-wave structure. For phase velocity measurements, as for example obtained by a refraction line field array, the differences in phase arrival times for traces obtained at different times can be measured directly and compared. Because shifts in phase arrival times are cumulative along the propagation path, tomography could also be applied to differential phase velocities. This research will focus on developing the differential techniques for the refraction line field arrays because the data sets are smaller, require less processing and are less susceptible to uncertainties in image resolution introduced by tomography. In particular, we consider an arrangement where the linear array of geophones can be located over a zone of induced anomalous fluid content. Application to more complex data acquisition arrays would be easier once the techniques are perfected for simpler acquisition geometry.

The theory for differential surface wave analysis along a line was presented in Long and Toteva (2005). Following that paper, the theory has undergone many refinements and changes. The current status of the analysis technique is outlined below. 
Problems encountered: The problems encountered were mainly associated with data acquisition and the details of instrumental response. The modifications and changes in analysis were largely designed to minimize acquisitions problems. To a large extent, the differential analysis techniques are so sensitive, that subtle variations in source excitation and origin time can introduce significant scatter in the interpreted phase arrival times. Modifications, which are outlined below, include separate determination of source time and frequency response.

There was no significant departure from the planned methodology. Most of the changes could be characterized as refinements to a general analysis method and each has let to significant improvements in the anaysis.

Test Sites: Data were obtained at two sites. The Georgia Southern University Research Well Field, and the Panola Mountain Research Watershed.

The Georgia Southern University Well Field consists of three well nests, each with a shallow and deep well penetrating from 17 to $28 \mathrm{ft}$ and 78 to $87 \mathrm{ft}$, respectively, into Coastal Plain sediments. This site will allow pumping of water in an area where the rocks are porous and the effect can be measured at distance. It would also allow testing the technique with deeper-penetrating lower-frequency surface waves.

The most significant field tests and data are likely to come from measurements in the US Geological Survey, Panola Mountain Research Watershed. This is a complete small watershed in which extensive arrays of water monitoring equipment have been set up. The objective is to observe variations in water content in soil and variations in runoff rates following storm events. We have established two arrays by setting out permanent placements for seismic sensor that will allow exact reoccupation of recording sites. One line is parallel to the streambed and will be used to measure ground water levels adjacent to the stream. The second is perpendicular to the streambed and extends up hill in an area where the ground is covered by water level monitors. The area is unique in that Panola Mountain is an exposed outcrop on granite gneiss. In the watershed, the granite gneiss is covered with a relatively thin layer of soil and weathered rock. Hence, during storm events, the soil can become rapidly saturated, and the draining of that soil over a period of a few days can be used to obtain varying conditions of ground water in a way that could not be repeated using pumping in wells. The proposed extension to this project will allow measurement of more than one significant rain event. The third array is set up on the outcrop and will be used to test the capability of seismic methods to image fractures and to image variations in fracture reflectivity with variations in water saturation. A forth test site in the Panola Mountain Research Watershed was located over a well with known distribution of fractures at depth. This data was also related to detecting scattered waves from fractures.

Computational Tools: Data acquisition and data analysis techniques have been developed and expanded over the first year and a half. During the course of this study, we have developed a portable computer-based data acquisition program. The geophones and amplifiers are standard commercial units, but the acquisition is 16-bit 16-channel digitizer with computer programs. We acquire and stack, as appropriate, the data to get either individual or averaged traces. The acquisition program will then save the data in 
its own format, or in conventional formats including SU and SEGY. The program has the capability of adjusting gain for individual channels, adjusting the sequence of channels and performing any adjustment that might be necessary for comparison of successive data sets, including multiple signal averaging.

For the analysis, a new program was developed that allows direct comparison of sets of seismic traces. This differential analysis package is set up to allow adjustment of the relative amplitudes of traces and to allow time shifting of the traces. We have discovered that the analysis is sensitive to minute differences in the source origin or trigger time. This program allows simultaneous examination of time shifts and amplitude changes in the recorded data and allows saving the difference data for further analysis.

The rest of the analysis has been performed using easily editable MATLAB programming techniques.

Results: The general results of this project are in preparation for presentation at SAGEEP in April, 2006. The following is a draft version of the paper to be submitted.

\section{Introduction}

For nearly 50 years, surface waves that propagate through near-surface soils and rocks have been utilized in engineering for the determination of the small-strain dynamic properties of soils and rocks (Okada, H., 2003). In recent years, techniques like SASW (Stokoe II, K.H. and others, 1989) and MASW (Xia, J., and others, 2000) have improved the interpretation capability and speed of acquisition. Rayleigh waves are most often used in these studies because the shear wave velocity primarily determines their velocity and because they are the largest amplitude wave excited by a surface source. Long and Toteva (2005) presented theoretical techniques for examining temporal variations in shear-wave velocity in shallow soils using surface waves. This paper further develops the concept of using surface wave velocities to monitor temporal variations in ground water by presenting three field examples.

The technique described in this paper uses Rayleigh waves to measure subtle temporal changes in the phase velocity of surface waves. We examined three types of perturbation: those introduced by various levels of saturation before and after significant rain events, those caused by the injection of water, and those caused by pumping water from a well. The measurement of temporal velocity perturbations has undergone significant development over the last decade and is known as time-lapse analysis or, when applied to 3D imaging, as 4D seismic processing in the oil industry. Recently, Vasco et al. (2004) have used 4D seismic to directly image reservoir flow properties. We develop this concept for seismic experiments that use surface waves to measure changes in the upper few meters of soil and rock. The basis for this technique is that water penetration in the soil should change the state of stress as well as the physical properties of the rock and these changing conditions would have a detectable effect on the shear wave velocity. Such changes would lead to a change in the phase velocity of the Rayleigh waves. In this study, we use reference traces that are recorded before perturbation and/or from areas not affected by the perturbation of the ground water and 
measure time delays observed on data that are recorded after perturbation of the ground water.

We designed our experiments and analysis to maximize repeatability of the measurements. All geophones and the source were placed at the same location before and after perturbation of the ground water. For some data, this required compensation for differences in the source function. Our results show measurable changes in the phase velocity due to the perturbation of the water table. Although such changes are not easily observed on the dispersion curve using conventional techniques, we found that differential methods could easily detect subtle changes.

\section{The Technique for Differential Analysis of Surface Waves}

The key to differential surface wave interpretation is recognizing that there exists a measurable sensitivity of shear wave velocity to variations in water content. The introduction or removal of fluids in soils changes the velocity by changing the state of stress as well as by changing the physical properties of the earth materials. Most observations of soil velocities at shallow depths suggest that they depend roughly on both composition and stress. Sands have a lower velocity than clay rich soils. The main contributors to the velocity increase with depth are the increased lithostatic stress and resulting increase in consolidation. Surface wave velocities in the areas of our study were in the range of 100 to $400 \mathrm{~m} / \mathrm{s}$. The penetration of fluids into a soil would cause a change in the shear-wave velocity on a local scale. The mechanisms are very likely a complex combination of immediate stress effects and longer term changes in material properties induced by changes in water content. We hypothesize that these changes could measurably affect the shear wave velocity.

The close relationship of the Rayleigh wave phase velocity to the shear wave velocity suggests that surface waves could measure changes in water content, and the dispersion properties of the Rayleigh waves could be used to identify the depths at which perturbations in water content is affecting the shear-wave velocity. For interpretation of perturbations in the structure we use measurements of the phase velocity. The phase shift or phase perturbation at any point from the seismic source is a cumulative function of phase velocity perturbations between the source and that point. We are interested in directly observing differences in phase velocity and must first find the perturbations in phase velocity. The travel time, $\boldsymbol{t}$, of a phase at given frequency traveling from a reference or start position, $\boldsymbol{x}_{\text {ref }}$, with phase, $\varphi\left(x_{\text {ref }}\right)$ to the observed position at distance, $\boldsymbol{x}$, with phase, $\varphi(x)$. under the condition that the phase velocity, $c(x)$, is a function of position can be expressed as:

$$
t=\frac{\phi(x)-\phi_{r e f}(x)}{\omega}=\int_{x_{r}}^{x} \frac{d x}{c(x)}
$$

A similar equation can be written for the travel time, $\boldsymbol{t}^{\prime}$, for a phase in a perturbed velocity structure by expressing the perturbed velocity as, $\boldsymbol{c}(\boldsymbol{x})+\boldsymbol{\Delta c}(\boldsymbol{x})$. The difference in these two times is then measured directly by the difference in the phase spectra of the arrivals and the difference in the integrals. In this application, $\boldsymbol{\Delta} \boldsymbol{c}(\boldsymbol{x})$ is small and will vary about zero when compared to a relatively large and positive value for $\boldsymbol{c}(\boldsymbol{x})$. Thus, 
we can use the mean value theorem to pull $\boldsymbol{c}(\boldsymbol{x})$ out of the integral. For each frequency the integral equation according to Long and Toteva (2005) that has to be solved is:

$$
\Delta t=\frac{\Delta \phi(\omega, x)}{\omega} \approx \frac{1}{\bar{c}(\omega)^{2}} \int_{0}^{x} \Delta c(\omega, x) d x
$$

where $\Delta \mathrm{c}(\omega)$ is the change in average phase velocity from the shot point to the geophone at $\mathrm{x}, \Delta \phi(\omega)$ is the phase shift and $\Delta \mathrm{t}$ is the time shift measured over the total distance.

This is an integral equation that can be solved either by differentiating the observed phase differences with respect to distance, or by solving a numerical approximation. However, in this study we are primarily concerned with computing $\boldsymbol{\Delta} \boldsymbol{t}$ for paths from the origin to the geophone in order to assess the capability of identifying perturbations in velocity.

In Long and Toteva (2005) we presented a technique appropriate for interpreting perturbations in phase velocity along a line of geophones. That technique was based on a trace developed by taking the difference between two traces in the time domain before converting to the frequency domain. In this paper we present an analysis technique for measuring travel time perturbations for the phase arrivals at single stations. The objective of this reduction is to correct, if appropriate, for noise in the event trigger and for variations in the frequency content of the source. The technique also provides a means to isolate specific phases in the seismogram for analysis.

Step 1 is a correction for errors in the event trigger time. The differential analysis detects variations in travel time of less than a millisecond. We have found that scatter in the trigger time can introduce variations on the order of a millisecond, equivalent to six sample points. To remove this scatter, we cross-correlated the first P-wave arrival at stations minimally affected by the area of ground water perturbation in soil velocity.

Step 2 is to compute and apply a source frequency content correction factor. This operation is performed in the frequency domain and applied to the entire trace. We choose shots before and after perturbation for a path that is not strongly influenced by the water perturbation. We also have to assume that the source is does not have significant variation with azimuth. For this path, we assume that the path effects will cancel out and the ratio of the spectra of the two traces should approximate a correction factor needed to convert the source after the introduction of a ground water perturbation into the source before the perturbation. We assume that the impulse response of the earth for this unperturbed identical path should be the same and any difference in the records would be derived from differences in the source. This correction was minimal when using the controlled weight drop, but provided significant improvement when applied to the sledgehammer shots.

Step 3 is to apply a zero phase Gaussian filter to isolate a selected frequency band and transform that back into the time domain. Step 3 is analogous to multiple filter technique applied to surface waves.

Step 4 is to identify the arrival time of the surface wave at the center frequency for the Gaussian filter. We then apply a tapered window centered on the target group arrival. The same window is used for the before and after traces. The windowed traces are transformed back into the frequency domain.

Step 5 is to compute the phase shift directly from the differences in the phase of the spectra and relate the phase shift to change in time according to equation 2. 
These phase shifts and delay times are total system responses including the influence of converted waves and background noise as well as the dominant effect of changes in phase velocity of the surface waves.

\section{Test site and Data Acquisition}

Test sites

For this study we obtained data at two sites. The first was the Panola Mountain Research Watershed (PMRW) and the second was a research well on the campus of Georgia Southern University (GSU). At the PMRW, the seismic recording line was parallel to and in the flood plane10 meters away from a stream that drains the watershed. The flood plane is narrow at this point and the ground surface slopes up approximately 20 degrees from the line. The near-surface soil is a mix of accumulated humus and weathered Panola granite. The total thickness under the line was on the order of 5 meters. The soil layer thins moving upslope from the seismic recording line approximately 50 meters to where the Panola Granite is exposed at the surface. The water table was 1.5 meters below the surface. The GSU site is located in the coastal plane and is in an area of sand rich soils of unknown thickness. The site was in a level portion of a flood plane for a stream located 20 meters from the study area. Both areas were forested.

\section{Experimental Setup}

At the PMRW site we used 16 conventional $15 \mathrm{~Hz}$ geophones that were placed at 1-meter intervals along a straight line (Figure 1). To assure repeatability of the experiment and to eliminate variations in instrument plant and response, we constructed cement mounts for the geophones and all tests in 2005 were taken with he same permanently placed geophones. A water level monitor was located one meter down slope from the line of seismometers. Two 3-inch PVC pipes that extend approximately 1 meter into the soil were available and located $30 \mathrm{~cm}$ upslope from the seismometer array. These were near seismometers 7 and 9 and were used in this study to put water into the ground.

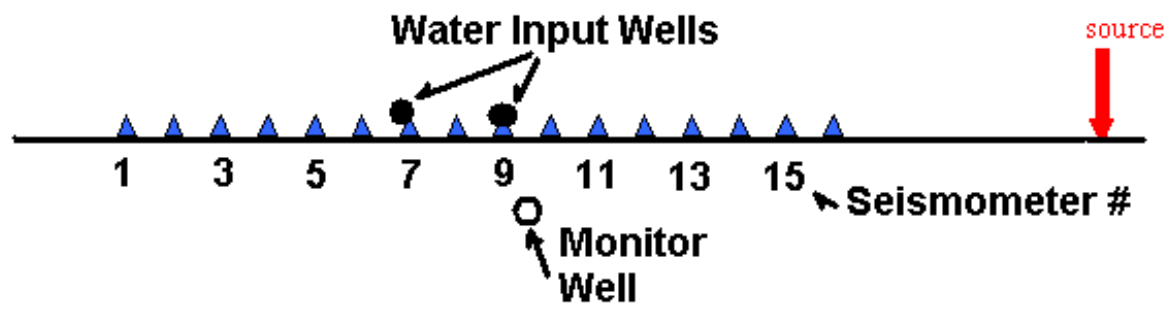

Figure 1: Geophone array at Panola Mountain Research Watershed. 
A weight drop source (Long and Toteva, 2005) was designed to produce a repeatable impulse for the source wavelet. It is constructed as a hollow cylinder with diameter of about 0.11 meters and height of 0.32 meters. The cylinder slides down a 1inch $(2.54 \mathrm{~cm})$ diameter pipe a distance of 0.82 meters. Elastic cords were used to accelerate the source. Data obtained from this array consisted of sets of records obtained during extended dry periods and following two major rain events, hurricanes Frances and Jeane. In addition, we obtained continuous records at one-minute intervals during injection of water along the seismic recording line. The seismic recording array at the GSU site was a rectangle (Figure 2.) designed ultimately for tomography, the first step of which is the determination of anomalous travel times. Data were recorded on two adjacent sides and the sources were placed on the opposite sides. At the GSU site, a sludge hammer and wood block were used for a source. We used a wood block for impact for surface waves in order to generate energy at lower frequencies, those appropriate for the surface waves of interest. Two sets of data were obtained. The first was obtained before any perturbation of the groundwater. The second was obtained after about 20 minutes of pumping and a lowering of the water level by 5 feet to 13.75 below the ground surface. The water level before pumping was at 8.75 feet below the surface. At the end of the second set of data the ground water level in the well had recovered only 0.85 feet to a depth of 12.9 feet.

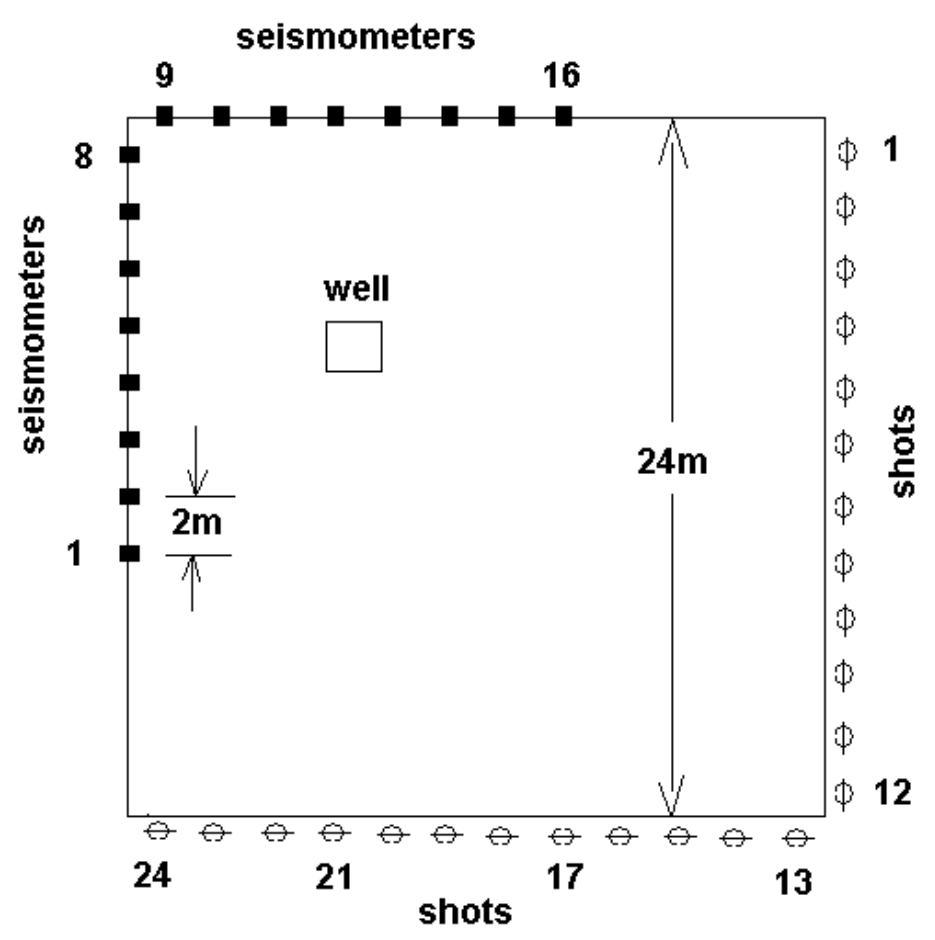

Figure 2: Layout of Geophones and Sources at the Georgia Southern University site. 


\section{Data analysis}

For the three data sets obtained at the PMRW during periods of differing groundwater saturation, the data analysis started with choosing records that showed good repeatability of the shape of the source function. Comparison of the consistent records showed significant differences in phase arrival and amplitude between the dry records and records taken after the rain events. Figure 3 shows the comparison between data during dry conditions and after hurricane Frances. While there exists a general similarity in the traces and the source functions were very similar, the differences were easily observed. Figure 4 shows the comparison for traces following the two rain events. These, again, are generally similar but with easily observed differences in the signature.

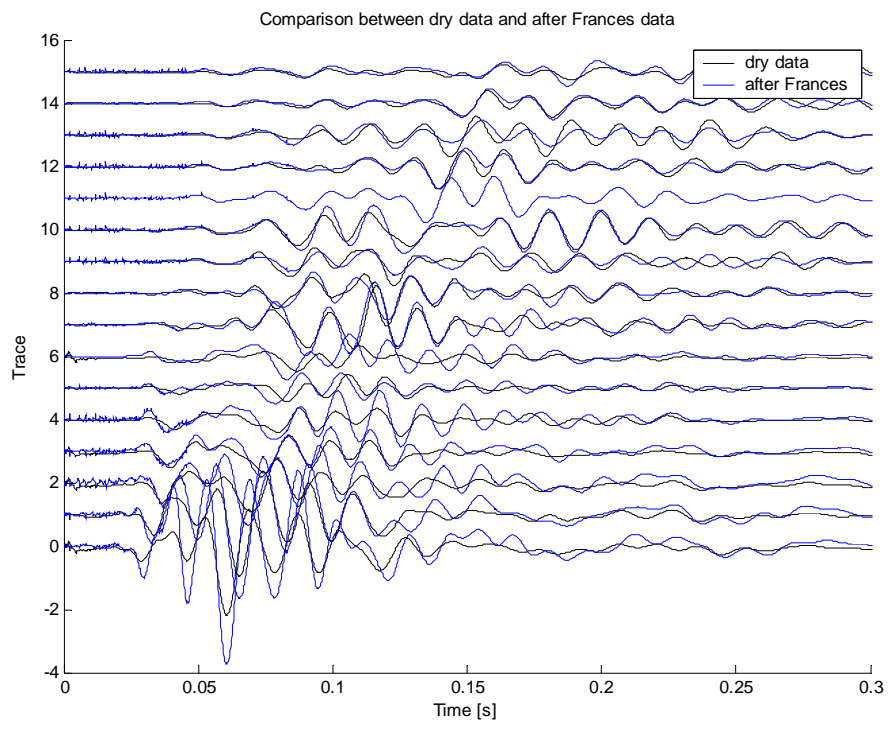

Figure 3: Comparison of dry conditions with data obtained after hurricane Frances.

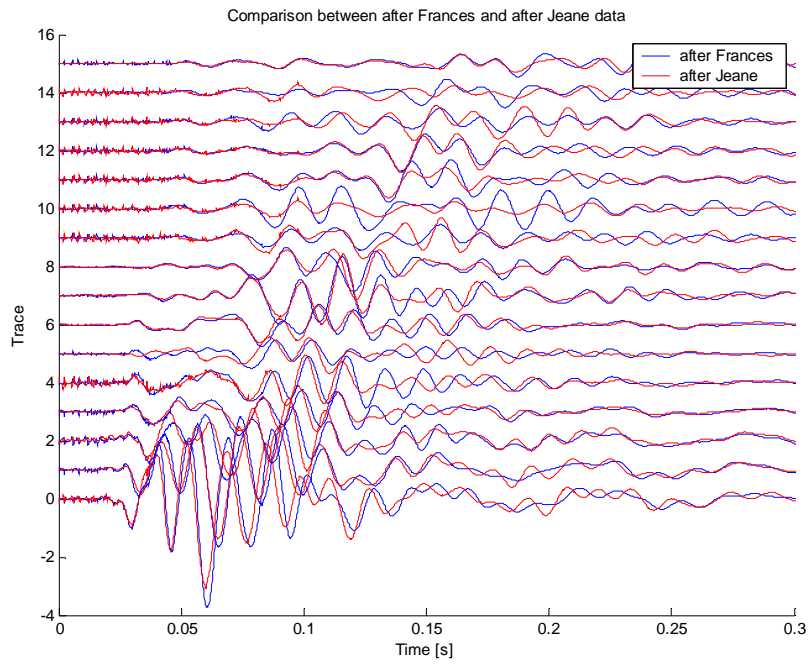

Figure 4: Comparison of traces after two rain events. 
The differences in the traces suggested that a conventional analysis could distinguish among the three sets of data. Figure 5 demonstrates a multiple filter analysis for group velocity for the three data sets. In all tests we showed that the tests over a short time period were repeatable, giving in all cases almost identical signatures. The differences represent variations in the velocity structure with time. In order to quantify these changes, we have computed group velocities from the data and for filtered data we have computed the phase shifts. In the 20 to 30 Hertz range, which is appropriate for a reasonable sampling of the top meter of soil and rock, the velocities following the two hurricanes were distinctly lower. Reduced velocity would be expected for the increased density associated with a higher water table.

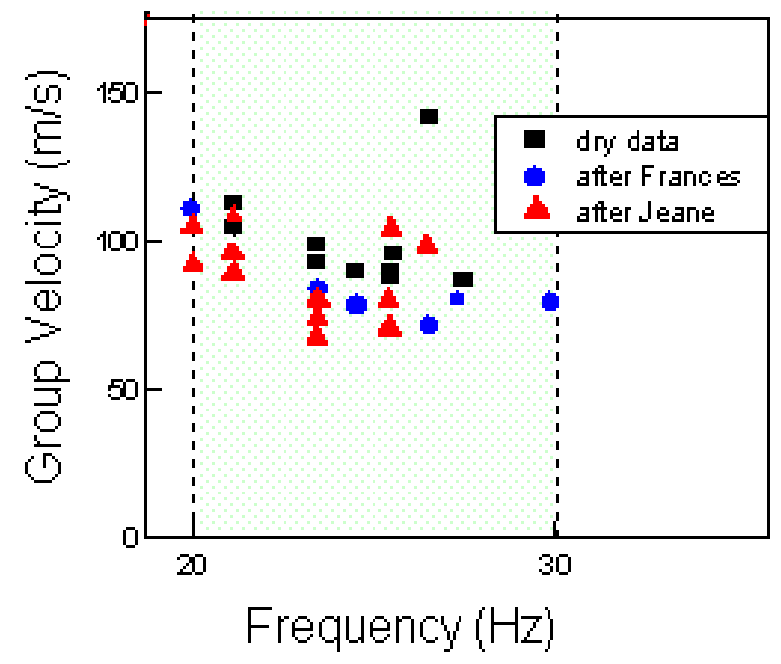

Figure 5: Group velocities observed before and after major storms and associated changes in water saturation.

In order to compute actual changes in arrival times for particular phases, we used a multiple filter technique. The filtered data for $30 \mathrm{~Hz}$ is shown in Figure 6 . The traces showed that the amplitudes were higher when water content was higher than they were during dry periods. While the phase shifts for the hurricane data were similar, there were significant shifts in phase relative to the dry recording periods. Figure 7 shows the computed time differences in the arrival of various phases. The reference is the record during the dry period. The dry trace line in Figure 7 indicates that the source is stable in the first half of the line and becomes increasingly unstable at the most distant geophones. The progressive increase in time in the short distances, with increasing times with distance, suggests that the velocity has been reduced by increased water content. The velocity decrease amounts to about 5 percent. 


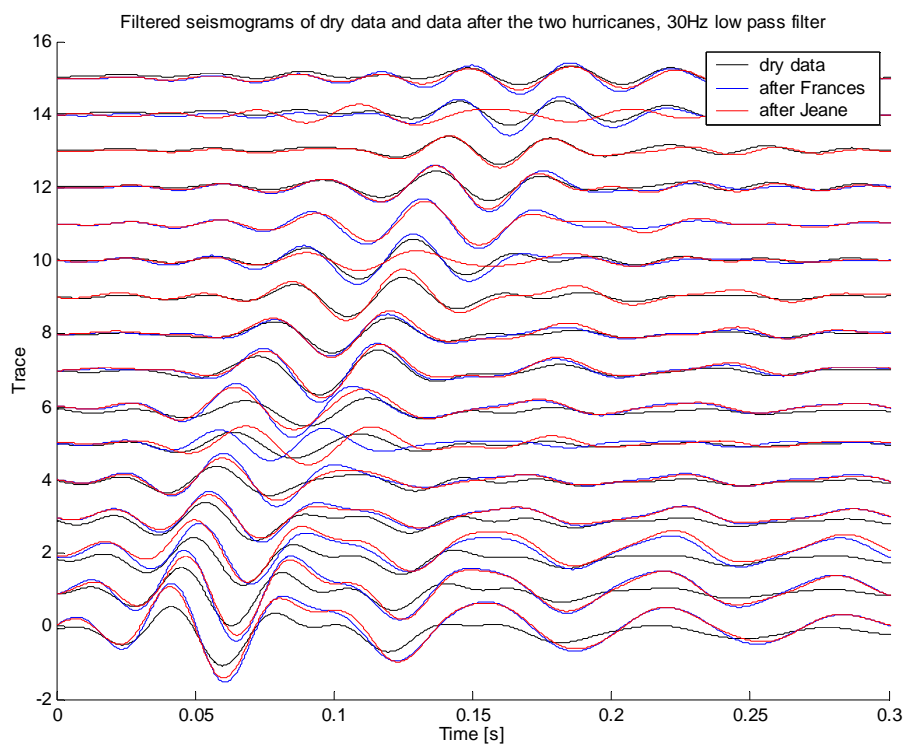

Figure 6: Seismograms filtered at $30 \mathrm{~Hz}$.

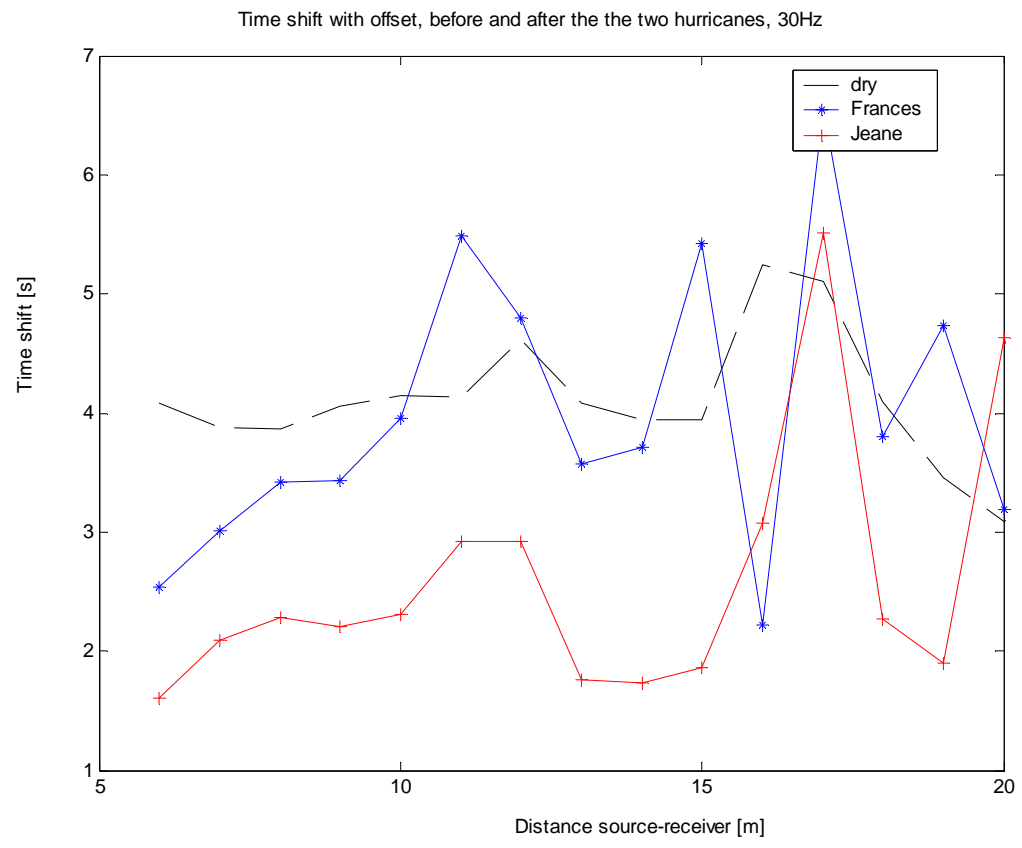

Figure 7: Computed time shifts for 30 Hertz.

Seismic records were obtained in 1-minute intervals at the PMRW during a water injection experiment. In this data set the delays were measured for a prominent surface wave phase. Figure 8 shows some results from this study. Water was injected into two wells after 3 minutes and up to 19 minutes into the measurements. Most of the water was put into the ground near geophone position 9 in Figure 1. The water level in the monitoring well at 20 minutes was raised 1.0 inch. At 40 minutes it had dropped back to an increase of only 0.1 inch. Trace 9 and 7 bracket the zone expected to be most affected 
by the increase in water. Trace 3 records waves that sample most of the perturbed area. Trace 12 was used as a reference trace, assuming that it was only slightly affected by the water perturbation. Trace 12 is 3 meters from trace 9 and 9 meters from the source.

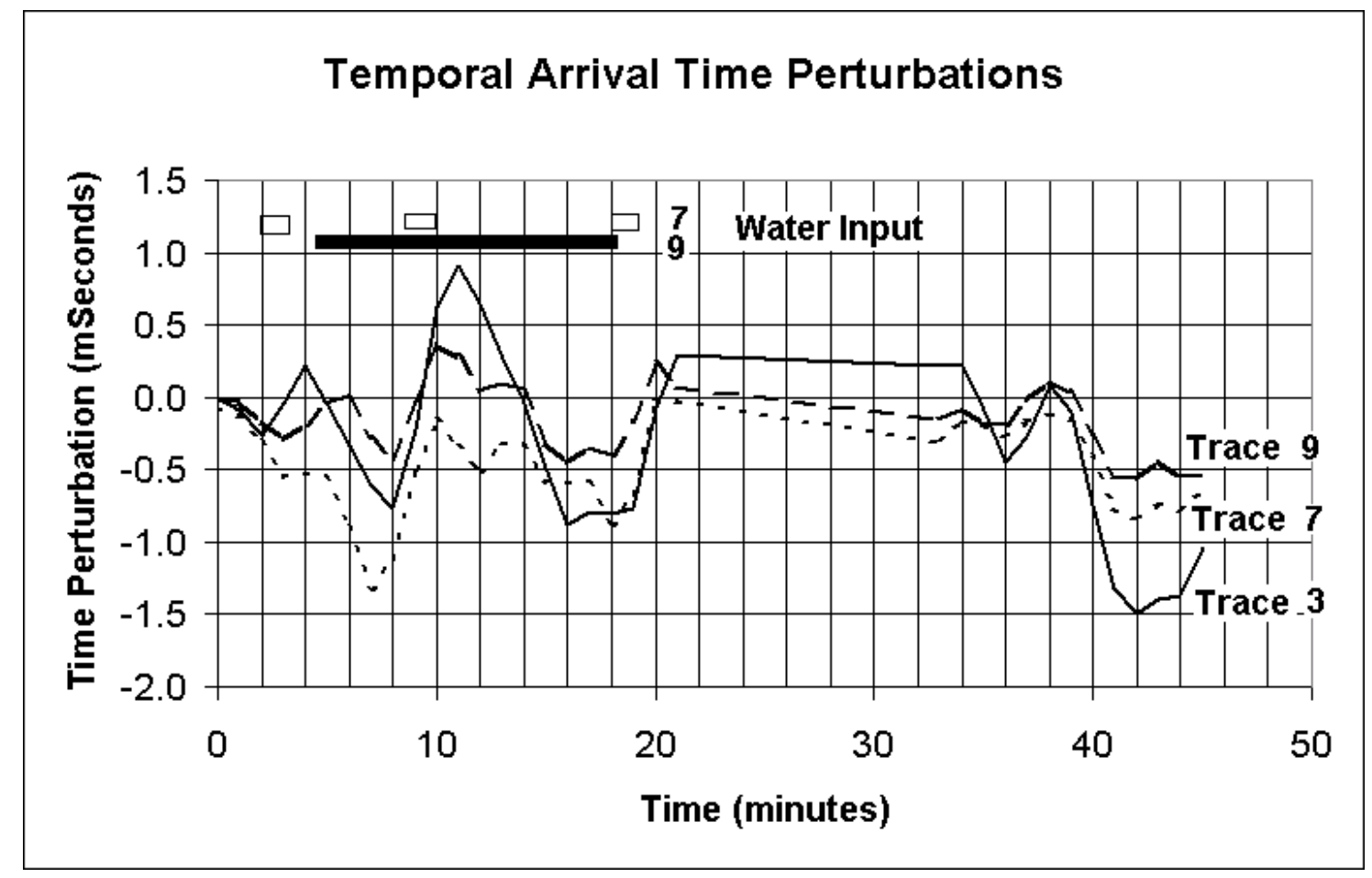

Figure 8: Temporal arrival time perturbations induced by water injection near the middle of a seismic recording line.

The data for the research well at GSU showed distinct decreases in velocity for waves going through the area affected by the 5 foot pumping down of the water in the well. Figure 9 shows one analyzed record for two shot points. The analysis here is completely as outlined above. That is corrections for origin time and source spectra were applied and the time shift for a selected portion of the surface wave was analyzed. The best response was for frequencies of 15 to $25 \mathrm{~Hz}$. Frequencies higher or lower, which are of low amplitude or not sufficiently dispersed are less consistent and tend to have much greater uncertainty. For frequencies of 15 to $25 \mathrm{~Hz}$ in figure 9, the arrivals near the well shadow are delayed up to 2 milliseconds. In this case a drawing down of the water table measurably decreased the velocity. 


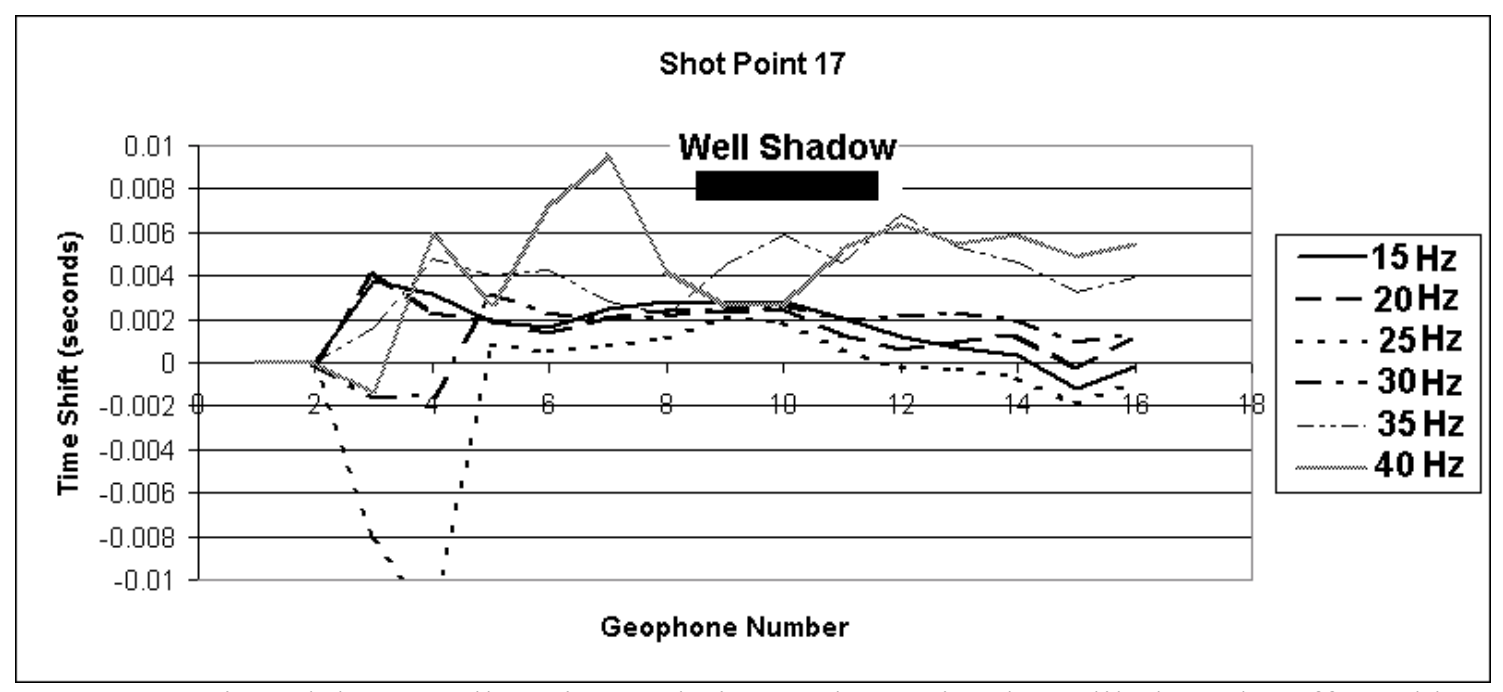

Figure 9. Time delays at all stations relative to the station least likely to be affected by pumping out the water.

\section{Discussion}

The data from the PMRW short time injection test demonstrated that the effects of water injection can come about rapidly in comparison to the 1 minute sample interval. The Figure 8 represents the response to 16 gallons of water put into the ground over a 14minute period. Figure 8 demonstrates that perturbations in arrival time up to 1.0 millisecond can be induced. These compare to the 2-millisecond variations interpreted for measurements during wet and dry periods. The transient character of the time delays is evident in Figure 8. During the water injection phase, trace 7 and trace 3 which most directly sample the perturbed area have the strongest response. However, with time the short-term response at trace 7 is attenuated and trace 3 has the greatest response, presumably because the water perturbation has spread out and affects a larger area. The measurement after storm events suggests that the velocity decreases with increased water content. The short time injection test shows a trend toward increasing velocity over the test period and transient short-term decreases during the injection phase.

\section{Conclusions}

Short and long term modifications to the phase velocity of surface waves cause measurable delay times on the order of milliseconds. The response is relatively rapid, less than a minute for velocity variations near points of injection. These variations appear to correlate with the time and location of perturbations in the water content of soils and may lead to methods to track movement of fluids in soil.

\section{References}

Long, L.T. and T. Toteva, 2005, Differential Surface Wave Detection of Changes in Fluid Saturation in Soils, in Proceedings of the Symposium on the Application of Geophysics to Engineering and Environmental Problems (SAGEEP), The Environmental and Engineering Geophysical Society April 2005 
Okada, H., 2003, The microtremor survey method, Society of Exploration Geophysicists, Tulsa, Oklahoma, 135p.

Stokoe II, K.H., Rix, G.L., and Nazarin, S., 1989, In situ seismic testing with surface wave, Proceedings of XII International Conference on Soil Mechanics and Foundation Engineering, pp.331-334.

Vasco, D. W., Datta-Gupta, A., Behrens, R., Condon, P., and Rickett, J., 2004, Seismic imaging of reservoir flow properties: Time-lapse amplitude changes, Geophysics, Vol. 69, No. 6, pp1425-1442.

Xia, J., Miller, R., Park, C., Hunter, J., and Harris, J., 2000, Comparing shear wave velocity profiles from MASW with borehole measurements in unconsolidated sediments, Fraser River Delta, B.C., Canada, Journal of Environmental and Engineering Geophysics, Vol.5, No.3, pp1-14.

\section{Acknowledgements}

This study was supported by the Office of Science (BER), U.S. Department of Energy, Grant No. DE-FG02-04ER63866. 


\title{
TECHNOLOGY TRANSFER ACTIVITIES.
}

\author{
Journal publications and expanded abstracts.
}

Long, L.T. and T. Toteva, (2005), Differential Surface Wave Detection of Changes in Fluid Saturation in Soils, in Proceedings of the Symposium on the Application of Geophysics to Engineering and Environmental Problems (SAGEEP), The Environmental and Engineering Geophysical Society April 2005

Long, L.T. and T. Toteva, Differential Surface Wave Interpretation of Temporal Changes in Velocity. Expanded Abstract for Society of Exploration Geophysicists Annual Meeting, Salt Lake City, UT October 9, 2002.

Long, L.T., Group Velocity Inversion Using Synthetic Surface Waves, Society of Environmental and Exploration Geophysicists, "SAGEEP" annual meeting, Las Vegas, NV, February, 2002

\section{In preparation:}

Long, L.T. and T. Toteva, Surface wave monitoring of shallow water reservoirs, in Proceedings of the Symposium on the Application of Geophysics to Engineering and Environmental Problems (SAGEEP), The Environmental and Engineering Geophysical Society April 2006

Surface wave tomography used to monitor water well drawdown. (complete analysis of Georgia Southern University research well site data)

Surface wave monitoring of injection into a shallow aquifer at the Panola Mountain Research Watershed

Toteva and Long, Scattering inversion for fractures in un-weathered rock

\section{Talks at meetings}

Long, L.T. 2006. Developments in seismic imaging of near-surface soils. Atlanta Geological Society, Invited lecture, March.

Long, L. T., and Tatiana Toteva, 2005. Velocity Structures in Four Dimensions, Eastern Section, Seismological Society of America meeting, Memphis, TN, October 3, 2005.

Toteva, Tatiana, L. T. Long, and Lester Williams, 2005. Three-dimensional Imaging of Scattering on a Granite Outcrop, Panola Mountain, Georgia, Eastern Section, Seismological Society of America meeting, Memphis, TN, October 3, 2005.

Toteva, Tatiana, and L. T. Long. 2005 A scattering inversion experiment to identify fractures on a granite outcrop. American Geophysical Union Meeting, New Orleans, May, 2005.

Tatiana Toteva, and L.T.Long, 2004. A Differential Approach for Detecting Temporal Changes in Near-Surface Earth Layers, 76th Annual Meeting, Eastern Section Seismological Society of 
America, Virginia Polytechnic Institute and State University, Blacksburg, Virginia. October 31 to November 1, 2004.

Chen, Michael, 2004. Processing the Perturbation Method76th Annual Meeting, Eastern Section Seismological Society of America, Virginia Polytechnic Institute and State University, Blacksburg, Virginia. October 31 to November 1, 2004.

Toteva, Tatiana, and L.T. Long. Imaging of shallow fractures, Western American Geophysical Union Meeting, San Francisco, December 2003.

Long, L.T., EMSP workshop presentation, Differential surface wave interpretation of perturbations of shallow soil structure. Richland, Washington, May 6, 2003 